Pacific Journal of Mathematics

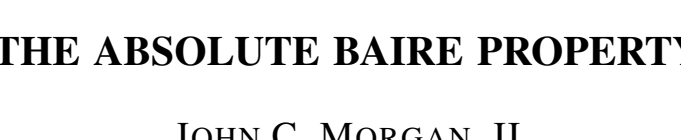




\title{
THE ABSOLUTE BAIRE PROPERTY
}

\author{
JohN C. MORGAN, II
}

In earlier papers the author has formulated an axiomatic foundation for a general theory of point sets, one of whose purposes is the unification of analogous theorems concerning Baire category and Lebesgue measure. Within this context, a method is given in the present paper for unifying the Baire property in the restricted sense and absolute measurability on the real line.

That absolute measurability is the appropriate measuretheoretic analogue of the Baire property in the restricted sense was suggested by $E$. Marczewski in a classical paper in 1937, after he had established the Baire property (in the wide sense) and Lebesgue measurability as analogous concepts.

Except for $\S \S 1$ and 5, $X$ will denote the real line. In $\S 1$ a brief review is given of basic definitions and facts from [6] which are pertinent to this paper. We define in $\S 2$ the "absolute Baire property" in terms of order preserving mappings. That this definition actually effects the desired unification is dependent upon the intimate relationship existing between perfect sets and sets of order type $\lambda$ (the order type of the real line) as discussed in $\S 3$. The classical examples are then presented in $\S 4$.

A central role in these investigations is played by certain families of perfect sets whose properties are given in $\S \S 5$ and 6 . In $\S 7$ we prove a general theorem which includes as special cases the known invariance under order isomorphisms of absolute measurability and of Marczewski's sets. Finally, some open problems are stated in $\S 8$.

\section{Preliminaries.}

Notation. If $\mathscr{S}$ is any family of sets, then the members of $\mathscr{S}$ will be called $\mathscr{S}$-sets.

Upon isolating properties common to the families of all closed intervals, all perfect sets, and all closed sets of positive Lebesgue measure, the following notion of a $\Omega$-family was obtained (see [6]).

Definition 1. A pair $(X, \mathscr{C})$ where $X$ is a nonempty set and $\mathscr{C}$ is a family of subsets of $X$ is a $\mathscr{R}$-family if the following axioms are satisfied.

1. $X=\mathrm{U} \mathscr{C}$.

2. Let $A$ be a $\mathscr{C}$-set and let $\mathscr{D}$ be a nonempty family of disjoint $\mathscr{C}$-sets which has power less than the power of $\mathscr{C}$. 
(a) If $A \cap(\bigcup \mathscr{D})$ contains a $\mathscr{C}$-set, then there is a $\mathscr{D}$-set $D$ such that $A \cap D$ contains a $\mathscr{C}$-set.

(b) If $A \cap(\mathrm{U} \mathscr{D})$ contains no $\mathscr{C}$-set, then there is a $\mathscr{C}$-set $B \subset$ $A$ which is disjoint from all $\mathscr{D}$-sets.

The symbol $\mathscr{C}$ will always signify a $\Re$-family, with respect to which the generalized Baire category concepts are defined.

Definition 2. A set $S \subset X$ is $\mathscr{C}$-singular if each $\mathscr{C}$-set $A$ contains a $\mathscr{C}$-set $B$ which is disjoint from $S$. We denote by $\mathscr{C}_{I}$ the family of all countable unions of $\mathscr{C}$-singular sets and by $\mathscr{C}_{I I}$ the family of all subsets of $X$ which are not $\mathscr{C}_{1}$-sets.

A set $S \subset X$ is a $\mathscr{C}_{I I}$-set everywhere on a $\mathscr{C}$-set $A$ if $S \cap B$ is a $\mathscr{C}_{I I}$-set for every $\mathscr{C}$-set $B \subset A$. The set $S$ is a $\mathscr{C}_{I I}$-set everywhere if $S \cap B$ is a $\mathscr{C}_{I I}$-set for every $\mathscr{C}$-set $B$.

We shall have occasion to use below the following fact concerning the intersection of two $\mathscr{C}$-sets (see [6] Theorem 1).

Proposition 1. If $A$ and $B$ are $\mathscr{C}$-sets, then either $A \cap B$ contains a $\mathscr{C}$-set or $A \cap B$ is $\mathscr{C}$-singular.

In addition, we will utilize the following generalization of a theorem of Banach (see [6] Theorem 2).

Fundamental Theorem. If $S$ is a $\mathscr{C}_{I I}$-set, then $S$ is a $\mathscr{C}_{I I}$-set everywhere on some $\mathscr{C}$-set.

Definition 3. A set $S \subset X$ has the Baire property with respect to $\mathscr{C}$ if for every $\mathscr{C}$-set $A$ there is a $\mathscr{C}$-set $B \subset A$ such that either $B \cap S$ or $B \cap(X-S)$ is a $\mathscr{C}_{I}$-set.

The family of all subsets of $X$ which have the Baire property with respect to $\mathscr{C}$ will be denoted by $\mathfrak{B}(\mathscr{C})$. This family is a $\sigma$ field containing the family $\mathscr{C}$ and the $\sigma$-ideal of $\mathscr{C}_{I}$-sets. Moreover, if $\mathscr{C}$ satisfies CCC (the countable chain condition), then $\mathfrak{B}(\mathscr{C})$ is closed under operation $(\mathscr{A})$.

Finally, we recall the notion of equivalence between $\Re$-families.

Definition 4. Two $\mathscr{R}$-families $\mathscr{C}$ and $\mathscr{D}$ of subsets of the same set $X$ are equivalent if $\mathscr{C}_{I}=\mathscr{D}_{I}$ and $\mathfrak{B}(\mathscr{C})=\mathfrak{B}(\mathscr{D})$.

2. The absolute Baire property. Let $\varphi$ be an order isomorphism mapping an ordered set $K$ onto an ordered set $L$. If $(K, \mathscr{C})$ is a $\Omega$-family and $\varphi(\mathscr{C})=\{\varphi(A): A \in \mathscr{C}\}$ then $(L, \varphi(\mathscr{C}))$ is a $\Re$-family 
with $\varphi(\mathscr{C})_{I}=\varphi(\mathscr{C} I)$ and $\mathfrak{B}[\varphi(\mathscr{C})]=\varphi[\mathfrak{B}(\mathscr{C})]$.

Definition 5. Assume $K \subset X$ has the relativized ordering of $X$ and $(K, \mathscr{C})$ is a $\Omega$-family. A set $S \subset X$ has the absolute Baire property with respect to $(K, \mathscr{C})$ (respectively, is an absolute $\mathscr{C}_{I}$-set) if $S \cap L$ has the Baire property (respectively, $S \cap L$ is a $\varphi(\mathscr{C})_{I}$-set) with respect to $(L, \varphi(\mathscr{C}))$ for every order isomorphic mapping $\varphi$ of $K$ onto a set $L \subset X$.

Notation. The family of all subsets of $X$ which have the absolute Baire property with respect to $(K, \mathscr{C})$ will be denoted by $\mathfrak{H}(\mathscr{C})$.

From the definition we immediately obtain the following facts.

1. If $K=X$ then $\mathfrak{A}(\mathscr{C})$ is a sub $\sigma$-field of $\mathfrak{B}(\mathscr{C})$ and the absolute $\mathscr{C}_{I}$-sets form a sub $\sigma$-ideal of $\mathscr{C}_{I}$.

2. If $\mathfrak{B}(\mathscr{C})$ is closed under operation $(\mathscr{C})$ then so also is $\mathfrak{A}(\mathscr{C})$.

3. If $(K, \mathscr{C})$ and $(K, \mathscr{D})$ are equivalent $\mathscr{R}$-families then $\mathfrak{X}(\mathscr{C})=$ $\mathfrak{A}(\mathscr{D})$.

3. The structure of perfect sets. Let $P$ be a bounded, nowhere dense, perfect set with $a=\inf P, b=\sup P$, and $\left(a_{1}, b_{1}\right),\left(a_{2}, b_{2}\right), \cdots$, $a<a_{n}<b_{n}<b, n=1,2, \cdots$, the intervals contiguous to $P$. Bendixson [1] has shown that upon removing the right hand side limit points $a, b_{1}, b_{2}, b_{3}, \cdots$ and the point $b$ from $P$ we obtain a set of order type $\lambda$. A similar result holds for any perfect set. As this fact is fundamental to this paper a proof is provided.

THEOREM 1. Every perfect set can be represented as a disjoint union of a set of order type $\lambda$ and a countable set.

Proof. It suffices to prove the theorem for bounded, perfect sets $P$.

Delete from $P$ the smallest and largest elements of $P$ and all right hand side limit points of $P$ (i.e., all points which are limit points from the right hand side but not from the left hand side). Only countably many points are thus removed. We show that the set $E$ of points remaining has order type $\lambda$. First we find a countable order-dense subset of $E$.

For each $x \in P$ associate a closed interval $I_{x}$ as follows:

(1) If $P$ contains a nondegenerate (i.e., nonempty interior) closed interval containing $x$ then let $I_{x}$ be the largest such closed interval.

(2) If $P$ contains no nondegenerate closed interval containing $x$ 
and every open interval containing $x$ also contains interior points of $P$ then let $I_{x}=\{x\}$.

(3) If $P$ contains no nondegenerate closed interval containing $x$ and there is an open interval containing $x$ which contains no interior points of $P$ then let $I_{x}$ be the largest closed interval $I$ containing $x$ such that $P \cap I$ is a nowhere dense perfect set.

For each $x \in P$, set $P_{x}=P \cap I_{x}$ and define a countable set $D_{x}$ in the following manner:

(i) If $P_{x}=[a, b], a<b$, then $D_{x}$ is the set consisting of all rational numbers in $(a, b)$ and the point $a$ if it was not deleted.

(ii) If $P_{x}=\{x\}$ then $D_{x}=\varnothing$.

(iii) If $P_{x}$ is a nowhere dense perfect set with $a=\inf P_{x}, b=$ $\sup P_{x}$ and contiguous intervals $\left(a_{1}, b_{1}\right),\left(a_{2}, b_{2}\right), \cdots, a<a_{n}<b_{n}<b$, $n=1,2, \cdots$ then $D_{x}$ is the set consisting of the points $a_{1}, a_{2}, \cdots$, the point $a$ if it was not deleted, and the point $b$ if it is not the largest element of $P$.

The set $D=\cup_{x \in P} D_{x}$ is a countable subset of $E$ which we now show to be order-dense in $E$.

Suppose $a, b \in E$ and $a<b$. Assume first that $a$ and $b$ both belong to $P_{x}$ for some $x$. If the interval $[a, b]$ is contained in $P_{x}$ then there is a rational number $c$ with $a<c<b$. If the interval $[a, b]$ is not contained in $P_{x}$ then $P_{x}$ is a nowhere dense, perfect set with contiguous intervals $\left(a_{1}, b_{1}\right),\left(a_{2}, b_{2}\right), \cdots$ and for some positive integer $n, a<a_{n}<b$. Next, assume $a \in P_{x}, b \in P_{y}$, and $x \neq y$. As $b$ is a left hand side limit point of $P$, there must be a point $c \in D_{x}$ such that $a<c<b$. Therefore $D$ is a countable order-dense subset of $E$.

Let $(A, B)$ be a Dedekind cut of $E$. Set $a=\sup A$ and $B=\inf$ $B$, then $a, b \in P$. If $a=b$ then, since no two-sided limit points were removed, we must have $a \in E$. Thus assume $a<b$. No points of $P$ will then lie in the interval $(a, b)$ and $b$ will be a right hand side limit point of $P$ so that $b \notin E$ and $a \in E$. The cut $(A, B)$ is thus seen to be determined by an element of $E$.

As $E$ also has no first or last element, $E$ has order type $\lambda$.

Notation. For any perfect set $P$ we denote by $\lambda(P)$ the set of order type $\lambda$ obtained by removing from $P$ its smallest and largest elements (if any) and all right hand side limit points of $P$.

Note that for perfect sets $A$ and $B$ we have $A \subset B$ if and only if $\lambda(A) \subset \lambda(B)$.

A set of order type $\lambda$ is a $\mathscr{G}_{-}$-set in $X$ which becomes a closed 
set upon adjoining a countable set of points (see [12], p. 133-134). Thus, by the Cantor-Bendixson theorem, the perfect sets and the sets of order type $\lambda$ are the same "modulo countable sets".

\section{Classical examples.}

EXAMPLE 1. Baire category. For each set $E \subset X$, let $\mathscr{R}(E)$ denote the family of all subsets of $E$ which have the classical Baire property relative to $E$, let $\mathscr{S}(E)$ denote the family of all subsets of $E$ which are of the first category relative to $E$, and let $\mathscr{B}(E)$ denote the family of all subsets of $E$ which are Borel sets relative to $E$.

A set $E \subset X$ is called "condensed-in-itself" if every point of $E$ is a condensation point of $E$.

Lemma 1. If $P$ and $Q$ are uncountable Borel sets condensed-inthemselves whose symmetric difference $P \Delta Q$ is countable then for every set $S \subset X$ we have

1. $S \cap P \in \mathscr{S}(P)$ if and only if $S \cap Q \in \mathscr{S}(Q)$.

2. $S \cap P \in \mathscr{R}(P)$ if and only if $S \cap Q \in \mathscr{R}(Q)$.

Proof. An elementary argument shows $S \cap P \in \mathscr{S}(P)$ if and only if $S \cap(P \cap Q) \in \mathscr{S}(P \cap Q)$ and $S \cap P \in \mathscr{R}(P)$ if and only if $S \cap$ $(P \cap Q) \in \mathscr{R}(P \cap Q)$. The lemma then follows upon interchanging $P$ and $Q$.

LemMa 2. If $L$ is a set of order type $\lambda$ then $\mathscr{B}(L)$ coincides with the $\sigma$-field $\mathscr{S}$ generated by all $L$-intervals of the form $(a, b) \cap$ $L$, with $a, b \in L, a<b$, and $(a, b)=\{x \in X: a<x<b\}$.

Theorem 2. If $(X, \mathscr{C})$ is the family of all open intervals $(a, b)$, $-\infty<a<b<+\infty$, then $\mathfrak{A}(\mathscr{C})$ is the family of all sets which have the Baire property in the restricted sense and the absolute $\mathscr{C}_{1}$-sets coincide with the sets always of the first category.

Proof. Let $P$ be any given perfect set, let $L=\lambda(P)$, and let $\phi$ be an order isomorphism of $X$ onto $L$. Define

$$
\mathscr{D}=\{G \cap L: G \text { is an open set in } X \text { and } G \cap L \neq \varnothing\} \text {. }
$$

Each $\varphi(\mathscr{C})$-set contains a $\mathscr{D}$-set and conversely. Hence the $\Re$ families $(L, \varphi(\mathscr{C}))$ and $(L, \mathscr{D})$ are equivalent, so that $\varphi(\mathscr{C})_{I}=\mathscr{D}_{I}=$ $\mathscr{S}(L)$ and $\mathfrak{B}[\mathscr{\varphi}(\mathscr{C})]=\mathfrak{B}(\mathscr{D})=\mathscr{R}(L)$. Applying Lemma 1, we see that if $S \subset X$ has the absolute Baire property with respect to $\mathscr{C}$ then $S \cap P \in \mathscr{R}(P)$ and if $S$ is an absolute $\mathscr{C}_{I}$-set then $S \cap P \in \mathscr{S}(P)$, 
for every perfect set $P$.

Conversely, let $\varphi$ be any order isomorphism of $X$ onto a set $L$ of order type $\lambda$ and let $P$ be the perfect kernel of the closure of $L$ in $X$. According to Lemma 1 , if $S$ is any subset of $X$ and $Q=$ $P \cap L$ then $S \cap P \in \mathscr{S}(P)$ implies $S \cap Q \in \mathscr{S}(Q)$ and $S \cap P \in \mathscr{R}(P)$ implies $S \cap Q \in \mathscr{R}(Q)$. We shall now show $S \cap Q \in \mathscr{S}(Q)$ implies $S \cap L \in \varphi(\mathscr{C})_{I}$ and $S \cap Q \in \mathscr{R}(Q)$ implies $S \cap L \in \mathscr{B}[\varphi(\mathscr{C})]$. It will then follow that if $S$ has the Baire property in the restricted sense then $S$ has the absolute Baire property with respect to $\mathscr{C}$ and if $S$ is always of the first category then $S$ is an absolute $\mathscr{C}_{I}$-set.

If $S \cap Q$ is nowhere dense relative to $Q$ then $S \cap Q$ is $\varphi(\mathscr{C})$ singular. Since $\varphi(\mathscr{C})_{I}=\varphi\left(\mathscr{C}_{I}\right)$ and every countable subset of $X$ is a $\mathscr{C}_{I}$-set, every countable subset of $L$ is a $\varphi(\mathscr{C})_{I}$-set. Therefore, if $S \cap Q \in \mathscr{S}(Q)$ then $S \cap L=(S \cap Q) \cup[S \cap(L-Q)]$ is a $\varphi(\mathscr{C})_{I}$-set. Assume next that $S \cap Q \in \mathscr{R}(Q)$, then $S \cap Q=A \cup N$ where $A \in \mathscr{B}(Q)$ and $N \in \mathscr{S}(Q)$. Hence $S \cap L=A \cup N \cup[S \cap(L-Q)]$, where $A \in \mathscr{B}(L)$ and $N \cup[S \cap(L-Q)] \in \varphi(\mathscr{C})_{I}$. By Lemma 2, $B \in \mathfrak{B}[\mathscr{\varphi}(\mathscr{C})]$ and consequently $S \cap L \in \mathfrak{B}[\varnothing(\mathscr{C})]$.

EXAmple 2. Lebesgue measure. If $E \subset X$ is a Borel set and $\mu$ is a measure on $(E, \mathscr{B}(E))$ then we shall denote by $\mathscr{C}(\mu)$ the family of all subsets of $E$ which are measurable with respect to the completion $\bar{\mu}$ of $\mu$, and by $\mathscr{N}(\mu)$ the family of all subsets of $E$ which are of measure zero with respect to the completion $\bar{\mu}$ of $\mu$.

$U$ will denote the open unit interval $(0,1)$ and $m$ will be Lebesgue measure on $U$.

Theorem 3. If $(X, \mathscr{C})$ is the family of all closed subsets of $X$ of positive Lebesgue measure then $\mathfrak{X}(\mathscr{C})$ consists of all absolutely measurable sets and the absolute $\mathscr{C}_{I}$-sets are the absolute null-sets.

Proof. It suffices to show the statement of the theorem is true when $(X, \mathscr{C})$ is replaced by the $\Re$-family $\left(U, \mathscr{C}^{\prime}\right)$, where $\mathscr{C}^{\prime}$ is the family of all closed subsets of $U$ of positive Lebesgue measure.

Let $\mu$ be a continuous probability measure on $(X, \mathscr{B}(X))$, let $P$ be the support of $\mu$, and let $L=\lambda(P)$. The restriction $\nu=\mu \uparrow \mathscr{B}(L)$ is a continuous probability measure on $(L, \mathscr{B}(L))$ which is positive on every $L$-interval of the form $(a, b) \cap L$, where $a, b \in L$ and $a<b$. Moreover, $\mathscr{N}(\nu)=\{S \cap L: S \in \mathscr{N}(\mu)\}$ and $\mathscr{L}(\nu)=\{S \cap L: S \in \mathscr{M}(\mu)\}$. Let $\psi$ be the order isomorphic mapping of $L$ onto $U$ defined by $\psi(x)=\nu(\{y \in L: y \leqq x\})$. For all $B \in \mathscr{B}(L)$ we have, as shown in [11], $\nu(B)=m[\psi(B)]$. Consequently $\mathscr{N}(\nu)=\psi^{-1}\left(\mathscr{C}^{\prime}\right)_{I}$ and $\mathscr{C}(\nu)=\mathfrak{B}\left[\psi^{-1}\left(\mathscr{C}^{\prime}\right)\right]$. Therefore, if $S \in \mathfrak{N}\left(\mathscr{C}^{\prime}\right)$ then $S$ is absolutely measurable and if $S$ is an absolute $\mathscr{C}_{1}^{\prime}$-set then $S$ is an absolute null-set. 
For the converse, let $\varphi$ be any order isomorphism mapping $U$ onto a set $L$ of order type $\lambda$. Let $\nu$ be the measure on $(L, \mathscr{B}(L))$ induced by $\varphi$ and Lebesgue measure $m$, i.e., $\nu(B)=m\left[\varphi^{-1}(B)\right]$ for each $B \in \mathscr{B}(L) . \quad \nu$ is a continuous probability measure which is of positive measure on every $L$-interval $(a, b) \cap L, a, b \in L, a<b$, and we have $\mathscr{N}(\nu)=\varphi\left(\mathscr{C}^{\prime}\right)_{I}$ and $\mathscr{C}(\nu)=\mathfrak{B}\left[\varphi\left(\mathscr{C}^{\prime}\right)\right]$. Extend $\nu$ to a continuous probability measure $\mu$ on $(X, \mathscr{B}(X))$, whose support is the perfect kernel of the closure of $L$ in $X$, by defining $\mu(B)=\nu(B \cap L)$ for each $B \in \mathscr{B}(X)$. Then $\mathscr{N}(\nu)=\{S \cap L: S \in \mathscr{N}(\mu)\}$ and $\mathscr{L}(\nu)=$ $\{S \cap L: S \in \mathscr{C}(\mu)\}$. It follows that if $S$ is absolutely measurable then $S \in \mathfrak{A}\left(\mathscr{C}^{\prime}\right)$ and if $S$ is an absolute null-set then $S$ is an absolute $\mathscr{C}_{1}^{\prime}$-set.

EXAMPle 3. Marczewski sets. A set $S \subset X$ is a Marczewski set (see [10]) if every perfect set $P \subset X$ contains a perfect set $Q$ such that either $Q \subset S$ or $Q \subset X-S$. The Marczewski sets are the sets which have the Baire property with respect to the $\Omega$-family of all perfect subsets of $X$. A set $S \subset X$ will be called a Marczwski singular set if it is singular with respect to this $\Re$-family, i.e., if every perfect set $P \subset X$ contains a perfect set $Q$ which is disjoint from $S$.

TheOREM 4. If $(X, \mathscr{C})$ is the family of all perfect subsets of $X$ then $\mathfrak{A}(\mathscr{C})=\mathfrak{B}(\mathscr{C})$ and the absolute $\mathscr{C}_{I}$-sets are the same as the Marczewski singular sets.

Proof. Suppose $S$ is a Marczewski set and let $\phi$ be an order isomorphism of $X$ onto a set $L$ of oredr type $\lambda$. If $A$ is any $\varphi(\mathscr{C})$ set then $A$ contains a perfect set $P$ which in turn contains a perfect set $Q$ such that $Q \subset S$ or $Q \subset X-S$. Moreover $Q$ contains a $\varphi(\mathscr{C})$ set $B$ such that $B \subset S \cap L$ or $B \subset L-(S \cap L)$, whence $S \cap L \in \mathfrak{B}[\varphi(\mathscr{C})]$. The set $S$ thus has the absolute Baire property with respect to $\mathscr{C}$. It is also clear that the Marczewski singular sets are absolute $\mathscr{C}_{I^{-}}$ sets.

5. S-families. Throughout this section $(X, d)$ will denote a complete, separable metric space with no isolated points.

Definition 6. A $\Re$-family $(X, \mathscr{C})$ consisting of perfect sets is called a $\mathfrak{P}$-family if it satisfies the condition

(+) for every $\mathscr{C}$-set $A$ and every point $x \in A$ there is a descending sequence $\left\langle A_{n}\right\rangle_{n=1}^{\infty}$ of $\mathscr{C}$-sets such that $x \in A_{n}, A_{n} \subset$ $A$, and $\operatorname{diam}\left(A_{n}\right) \leqq 1 / n$ for each $n$.

An equivalent condition is given in the next theorem. 
THEOREM 5. A necessary and sufficient condition that a $\Re$ family of perfect sets be a $\mathfrak{P}$-family is that for each $\mathscr{C}$-set $A$, each positive integer $n$, and each pair $x_{1}, x_{2}$ of distinct points in $A$, there exist disjoint $\mathscr{C}$-sets $A_{1}, A_{2}$ such that $x_{i} \in A_{i}, A_{i} \subset A$, and $\operatorname{diam}\left(A_{i}\right) \leqq$ $1 / n$ for $i=1,2$.

Unless otherwise indicated $(X, \mathscr{C})$ will hereafter denote a $\mathfrak{S}$ family.

Examples of $\mathfrak{3}$-families (see [5], [6], [9]).

4. Let $Q$ be a countable set dense in $X$ and let $\mathscr{C}$ be the family of all closures of open balls $\{x \in X: d(x, r)<1 / n\}, r \in Q, n=1,2, \cdots$.

5. Let $\mu$ be a continuous probability measure defined on the Borel subsets of $X$ and let $\mathscr{C}$ be the family of all closed sets which are of positive meaure in every neighborhood of each of their points.

6. Let $\mathscr{C}$ be the family of all perfect sets. We shall also denote this $\mathfrak{P}$-family by $\mathscr{P}$.

7. Assume the continuum hypothesis. Let $X$ be $n$-dimensional Euclidean space, let $h$ be a continuous function in $\mathscr{H}_{0}$, let $\mu^{h}$ be the Hausdorff measure associated with $h$, and let $\mathscr{C}$ be the family of all closed sets which are of positive $\mu^{h}$-measure in every neighborhood of each of their points.

8. Assume the continuum hypothesis. Let $X$ be $n$-dimensional Euclidean space and let $\mathscr{C}$ be the family of all closed sets which are of positive Hausdorff dimension in every neighborhood of each of their points.

Note that, when $X$ is the real line, the first three examples above are equivalent to the $\Re$-families of $\S 4$.

Definition 7. A $\Re$-family $(X, \mathscr{C})$, where $X$ is an arbitrary uncountable set, is called an \&-family if the following properties hold:

1. every $\mathscr{C}$-set is a $\mathscr{C}_{I I}$-set.

2. every countable set is a $\mathscr{C}_{I}$-set.

All theorems in [6] which are true for $\mathfrak{M}$-families are also true for \&-families. We also have

BAIRE'S THEOREM 6. Every $\mathfrak{P}$-family is an \&-family.

We next give some basic facts concerning $\mathfrak{P}$-families $(X, \mathscr{C})$.

THEOREM 7. If $A$ and $B$ are $\mathscr{C}$-sets whose symmetric difference 
$A \Delta B$ is a $\mathscr{C}_{I}$-set then $A=B$.

Proof. It suffices to show that if $A-B$ is a $\mathscr{C}_{1}$-set then $A \subset$ $B$. Assume $x \in A$ and let $\left\langle A_{n}\right\rangle_{n=1}^{\infty}$ satisfy condition (+). From the equality $A_{n}=\left(A_{n} \cap B\right) \cup\left(A_{n}-B\right)$ and the fact that $A_{n}-B$ is a $\mathscr{C}_{I^{-}}$ set, we conclude $A_{n} \cap B \neq \varnothing$ for every $n$. Therefore $x \in B$.

THEOREM 8. If $P$ is a perfect set which is not $\mathscr{C}$-singular then $P$ contains a $\mathscr{C}$-set.

Proof. If $P$ is not $\mathscr{C}$-singular then there is a $\mathscr{C}$-set $A$ such that $P \cap B \neq \varnothing$ for every $\mathscr{C}$-set $B \subset A$. It follows from condition (+) that $A \subset P$.

THEOREM 9. If $(X, \mathscr{C})$ is not equivalent to $(X, \mathscr{P})$ then there exists a $\mathscr{C}$-singular perfect set.

Proof. If there are no $\mathscr{C}$-singular perfect sets then every perfect set contains a $\mathscr{C}$-set. Hence every $\mathscr{C}$-set contains a $\mathscr{P}$-set and conversely, so $(X, \mathscr{C})$ is equivalent to $(X, \mathscr{P})$.

Corollary 10. If $(X, \mathscr{C})$ satisfies $\mathrm{CCC}$ then there exists a $\mathscr{C}$ singular perfect set.

THEOREM 11. Every Borel set has the Baire property with respect to $\mathscr{C}$.

Proof. Suppose $G$ is a nonempty open set and $A$ is any $\mathscr{C}$-set. If $A \cap G \neq \varnothing$ then there is a point $x \in G$ and a $\mathscr{C}$-set $B \subset A \cap G$ such that $x \in B$, whence $B \cap(X-G)=\varnothing$. It follows that every open set has the Baire property with respect to $\mathscr{C}$.

REMARK. In general, the $\sigma$-field generated by $\mathscr{C}$ will not contain all the Borel sets.

THEOREM 12. Every $\mathscr{C}_{I I}$-set with the Baire property contains a perfect set.

Proof. Let $S$ be a $\mathscr{C}_{I I}$-set with the Baire property and let $S$ be a $\mathscr{C}_{I I}$-set everywhere on a $\mathscr{C}$-set $A$. The set $A-S$ is then a $\mathscr{C}_{I}$-set, say $A-S=\bigcup_{n=1}^{\infty} S_{n}$ where each $S_{n}$ is $\mathscr{C}$-singular. A dyadic schema of $\mathscr{C}$-sets can be constructed in $A$ to determine a perfect set $P$ disjoint from every $S_{n}$. Thus $P$ is a perfect subset of $S$. 
COROLLARY 13. A set with the Baire property either contains a perfect set or its complement contains a perfect set.

For every $\mathscr{C}$-set $A$ the family $\mathscr{C} \uparrow A$ of all $\mathscr{C}$-sets contained in $A$ is a $\mathfrak{P}$-family, called "the restriction of $(X, \mathscr{C})$ to $A$ ".

THEOREM 14. If for every $\mathscr{C}$-set $A$ the $\mathfrak{P}$-family $(A, \mathscr{C} \uparrow A)$ is not equivalent to $(A, \mathscr{P} \uparrow A)$ then every $\mathscr{C}_{I I}$-set with the Baire property contains a $\mathscr{C}$-singular perfect set.

Proof. Assume $S$ is a $\mathscr{C}_{I I}$-set with the Baire property. By Theorem 12, the set $S$ contains a perfect set $P$. If $P$ is $\mathscr{C}$-singular then we are finished. Suppose then that $P$ is not $\mathscr{C}$-singular. By Theorem 8 , there is a $\mathscr{C}$-set $A$ contained in $P$. Since $(A, \mathscr{C} \uparrow A)$ is not equivalent to $(A, \mathscr{P} \uparrow A)$, we obtain from Theorem 9 the existence of a $\mathscr{C} \uparrow A$-singular perfect set $Q \subset A$. That $Q$ is also $\mathscr{C}$-singular is a consequence of Proposition 1.

CoROllaRY 15. If $(X, \mathscr{C})$ satisfies $\mathrm{CCC}$ then every $\mathscr{C}_{I I}$-set with the Baire property contains a $\mathscr{C}$-singular perfect set.

Definition 8 (cf. [6]). A set $S \subset X$ has property $(L)$ with respect to $\mathscr{C}$ if $S$ is uncountable and every uncountable subset of $S$ is a $\mathscr{C}_{I I}$-set.

THEOREM 16. If for every $\mathscr{C}$-set $A$ the $\mathfrak{P}$-family $(A, \mathscr{C} \uparrow A)$ is not equivalent to $(A, \mathscr{P} \uparrow A)$ then any set having property $(L)$ does not have the Baire property.

Proof. This is an immediate consequence of Theorem 14.

Corollary 17. If for every $\mathscr{C}$-set $A$ the $\mathfrak{P}$-family $(A, \mathscr{C} \uparrow A)$ is not equivalent to $(A, \mathscr{P} \uparrow A)$ then an uncountable set $S$ has property $(L)$ if and only if every subset of $S$ having the Baire property is countable.

A set which contains no perfect set and whose complement also contains no perfect set is called, following [8], a "Bernstein set."

THEOREM 18. A Bernstein set does not have the Baire property with respect to any $\mathfrak{B}$-family. In fact, any subset of a Bernstein set which has the Baire property with respect to a $\mathfrak{F}$-family $(X, \mathscr{C})$ is a $\mathscr{C}_{I^{-}}$set. 


\section{Proof. Apply Theorem 12.}

THEOREM 19. Every $\mathscr{C}_{I I}$-set has a subset which does not hav the Baire property. Equivalently, if every subset of $S$ has th Baire property then $S$ is a $\mathscr{C}_{I}$-set.

Proof. See the proof of Theorem 5.5 of [8].

CoRollary 20. If $(X, \mathscr{C})$ and $(X, \mathscr{D})$ are nonequivalent $\mathfrak{P}$ families and $\mathscr{C} \subset \mathscr{D}$ then there is a set which has the Baire property with respect to $\mathscr{C}$ but which does not have the Baire property with respect to $\mathscr{D}$.

Proof. Since $(X, \mathscr{C})$ and $(X, \mathscr{D})$ are not equivalent, there is a $\mathscr{D}$-set $A$ which contains no $\mathscr{C}$-set. Hence $A$ is a $\mathscr{D}_{I I}$-set and is $\mathscr{C}$-singular. Now apply Theorem 19.

6. The absolute Baire property with respect to a ß-family.

THEOREM 21. $\mathfrak{A}(\mathscr{C})$ is contained in the $\sigma$-field of Marczewski sets and the absolute $\mathscr{C}_{I}$-sets are contained in the $\sigma$-ideal of Marczewski singular sets.

Proof. Assume $S$ has the absolute Baire property with respect to $\mathscr{C}$. Let $P$ be any perfect set and let $\phi$ be an order isomorphism mapping $X$ onto $L=\lambda(P)$. If $S \cap L$ is a $\varphi(\mathscr{C})_{I I}$-set then by Theorem 12 there is a perfect set $R$ such that $R \subset \varphi^{-1}(S \cap L)$ and if $S \cap L$ is a $\varphi(\mathscr{C})_{I}$-set then there is a perfect set $R \subset X-\varphi^{-1}(S \cap L)$. Since $\varphi(R)$ is an uncountable Borel set, there exists a perfect set $Q \subset \varphi(R)$ with either $Q \subset S \cap L$ or $Q \subset L-S$. Hence $S$ is a Marczewski set. That the absolute $\mathscr{C}_{I}$-sets are Marczewski singular sets is now obvious.

As we have seen, for the family $\mathscr{P}$ of all perfect sets we have $\mathfrak{A}(\mathscr{P})=\mathfrak{B}(\mathscr{P})$. This property is characteristic of $\mathscr{P}$ in the following sence.

THEOREM 22. If $(X, \mathscr{C})$ is a $\mathfrak{P}$-family with $\mathfrak{A}(\mathscr{C})=\mathfrak{B}(\mathscr{C})$ then $(X, \mathscr{C})$ is equivalent to $(X, \mathscr{P})$.

Proof. Assume $\mathfrak{A}(\mathscr{C})=\mathfrak{B}(\mathscr{C})$ and $(X, \mathscr{C})$ is not equivalent to $(X, \mathscr{P}) . \quad$ By Theorem 9 there exists a $\mathscr{C}$-singular perfect set $P$. Every subset of $P$ has the Baire property with respect to $\mathscr{C}$ and hence, according to the preceding theorem, every subset of $P$ is a Marczewski set. Applying Theorem 19 we conclude $P$ is a Marczewski 
singular set!

Theorem 23. $2(\mathscr{C})$ contains all Borel sets.

Proof. It suffices to show $\mathfrak{A}(\mathscr{C})$ contains all open intervals. Let $\varphi$ be any order isomorphism of $X$ onto a set $L$ of order type $\lambda$. We show for every open interval $I$ and every $\varphi(\mathscr{C})$-set $A$ there is a $\varphi(\mathscr{C})$-set $B \subset A$ such that either $B \cap I$ or $B \cap(L-I)$ is a $\varphi(\mathscr{C})_{I}$-set.

If $A \cap I$ contains at most two points then $A \cap I$ is a $\varphi(\mathscr{C})_{I}$-set. Thus assume $A \cap I$ contains at least three points $a, b, c$ with $a<$ $c<b$. If $J$ is the open interval with endpoints $\varphi^{-1}(a), \varphi^{-1}(b)$ then there is a $\mathscr{C}$-set $E \subset \varphi^{-1}(A) \cap J$ such that $\varphi^{-1}(c) \in E$. Hence $B=\varphi(E)$ is a $\varphi(\mathscr{C})$-set contained in $A \cap I$ and $B \cap(L-I)=\varnothing$ is a $\varphi(\mathscr{C})_{I^{-}}$-set.

Corollary 24. If $(X, \mathscr{C})$ satisfies CCC then $\mathfrak{A}(\mathscr{C})$ contains all analytic sets.

Concerning the existence of uncountable absolute $\mathscr{C}_{I}$-sets we can easily generalize the theorem of Lusin and Sierpinski [4] to obtain

THEOREM 25. There exists a set $S \subset X$ of power $\aleph_{1}$ which is an absolute $\mathscr{C}_{I}$-set with respect to every $\mathfrak{B}$-family $(X, \mathscr{C})$ satisfying CCC.

7. On order isomorphic images of sets. In general the Baire property is not invariant under order isomorphisms. Specifically, we have

Theorem 26. If $(X, \mathscr{C})$ is not equivalent to $(X, \mathscr{P})$ then the Baire property with respect to $\mathscr{C}$ is not invariant under order isomorphisms.

Proof. By Theorem 9 there is a $\mathscr{C}$-singular perfect set $P$ and by Theorem 19 there is a set $S$ which does not have the Baire property with respect to $\mathscr{C}$. If $\varphi$ is any order isomorphism of $\lambda(P)$ onto $X$ then $\varphi^{-1}(S)$ has the Baire property and $S$ does not.

REMARK. If we remove from $\lambda(P)$ the dense set $D$ defined in the proof of Theorem 1 then the restriction of $\phi$ to the set $\lambda(P)-$ $D$ is an order preserving homeomorphism. Hence, if $(X, \mathscr{C})$ is not equivalent to $(X, \mathscr{P})$ then the Baire property with respect to $\mathscr{C}$ is not invariant under homeomorphisms. 
The remainder of this section is devoted to establishing, under certain conditions, the invariance of the absolute Baire property under order isomorphisms.

Without loss of generality, we can assume the space $X$ belongs to $\mathscr{C}$. For we can easily prove the following:

Lemma 3. If $(X, \mathscr{C})$ is a $\Re$-family (resp. a $\mathfrak{P}$-family) then $(X, \mathscr{C} \cup\{X\})$ is an equivalent $\Re$-family (resp. $\mathfrak{P}$-family).

Next, we associate with a $\mathfrak{P}$-family $(X, \mathscr{C})$ an equivalent $\Re$ family each of whose sets has order type $\lambda$.

Lemma 4. If $(X, \mathscr{C})$ is a $\mathfrak{P}$-family, with $X \in \mathscr{C}$, and $\mathscr{L}=$ $\{\lambda(A): A \in \mathscr{C}\}$ then $(X, \mathscr{L})$ is a $\Re$-family which is equivalent to $(X, \mathscr{C})$.

Proof. We first show $(X, \mathscr{L})$ is a $\Omega$-family. Axiom 1 is a consequence of the assumption $X \in \mathscr{C}$. Assume $\mathscr{C}=\left\{M_{\alpha}: \alpha<\Theta\right\}$, where $\Theta$ is a limit ordinal, is a nonempty family of disjoint $\mathscr{L}$-sets of power less than the power of $\mathscr{L}$, hence of power less than the power of $\mathscr{C}$, and let $L$ be an $\mathscr{L}$-set. For each set $S \subset X$ we denote the closure of $S$ by $\bar{S}$.

Suppose $L \cap\left(\bigcup_{\alpha} M_{\alpha}\right)$ contains an $\mathscr{L}$-set $K$, then $\bar{K} \cap\left(\bigcup_{\alpha} \bar{M}_{\alpha}\right)$ contains a $\mathscr{C}$-set. For, if $\bar{K} \cap\left(\bigcup_{\alpha} \bar{M}_{\alpha}\right)$ contains no $\mathscr{C}$-set then there is a $\mathscr{C}$-set $A \subset \bar{K}-\left(\bigcup_{\alpha} \bar{M}_{\alpha}\right)$ and hence $\lambda(A) \subset K-\left(\bigcup_{\alpha} M_{\alpha}\right)$, contradicting the fact that $\lambda(A) \subset \bigcup_{\alpha} M_{\alpha}$. Choose now $\alpha<\Theta$ so that $\bar{K} \cap \bar{M}_{\alpha}$ contains a $\mathscr{C}$-set $B$. Then $\lambda(B)$ is an $\mathscr{L}$-set contained in $L \cap M_{\alpha}$.

Suppose $L \cap\left(\mathrm{U}_{\alpha} M_{\alpha}\right)$ contains no $\mathscr{L}$-set. If $\bar{L} \cap\left(\mathrm{U}_{\alpha} \bar{M}_{\alpha}\right)$ contains a $\mathscr{C}$-set then there is an index $\alpha<\Theta$ such that $\bar{L} \cap \bar{M}_{\alpha}$ contains a $\mathscr{C}$-set $A$ and consequently $\lambda(A) \subset L \cap M_{\alpha} \subset L \cap\left(\mathrm{U}_{\alpha} M_{\alpha}\right)$. Therefore $\bar{L} \cap\left(\mathbf{U}_{\alpha} \bar{M}_{\alpha}\right)$ contains no $\mathscr{C}$-set and there is a $\mathscr{C}$-set $A \subset \bar{L}-\left(\mathrm{U}_{\alpha} \bar{M}_{\alpha}\right)$. Hence $\lambda(A)$ is an $\mathscr{L}$-set contained in $L-\left(\mathrm{U}_{\alpha} M_{\alpha}\right)$. Axiom 2 is thus satisfied.

To prove $(X, \mathscr{L})$ and $(X, \mathscr{C})$ are equivalent we first show $\mathscr{C}_{I}=$ $\mathscr{L}_{1}$. It is easily seen that if $S$ is a $\mathscr{C}$-singular set then $S$ is $\mathscr{L}$ singular. Therefore $\mathscr{C}_{I} \subset \mathscr{L}_{I}$. Suppose now that $S$ is an $\mathscr{L}$-singular set. If $A$ is any $\mathscr{C}$-set then there is an $\mathscr{L}$-set $K \subset \lambda(A)-S$ and it follows that $\bar{K} \cap S$ is countable. We have thus shown that for every $\mathscr{C}$-set $A$ there is a $\mathscr{C}$-set $B \subset A$ such that $B \cap S$ is a $\mathscr{C}_{I}$-set. By the Fundamental Theorem, $S$ is a $\mathscr{C}_{I}$-set. Therefore we have $\mathscr{L}_{I} \subset$ $\mathscr{C}_{1}$.

Assume $S$ has the Baire property with respect to $\mathscr{C}$ and suppose $S$ is an $\mathscr{L}_{I I}$-set everywhere on an $\mathscr{L}$-set $L$. Then for every $\mathscr{C}$-set 
$A \subset \bar{L}$, the set $A \cap S$ is a $\mathscr{C}_{I I}$-set. Hence there is a $\mathscr{C}$-set $B \subset \bar{L}$ such that $B \cap(X-S)$ is a $\mathscr{C}_{I}$-set. It follows that $\lambda(B)$ is an $\mathscr{L}$ set contained in $L$ and $\lambda(B) \cap(X-S)$ is an $\mathscr{L}_{I}$-set. Thus $S$ has the Baire property with respect to $\mathscr{L}$.

Conversely, assume $S$ has the Baire property with respect to $\mathscr{L}$ and suppose $S$ is a $\mathscr{C}_{I I}$-set everywhere on the $\mathscr{C}$-set $A$. Then for every $\mathscr{L}$-set $L \subset \lambda(A)$ the set $L \cap S$ is an $\mathscr{L}_{I I}$-set. Hence there is an $\mathscr{L}$-set $K \subset \lambda(A)$ such that $K \cap(X-S)$ is an $\mathscr{L}_{I}$-set. The set $\bar{K}$ is a $\mathscr{C}$-set contained in $A$ and $\bar{K} \cap(X-S)$ is a $\mathscr{C}_{I}$-set. Thus $S$ has the Baire property with respect to $\mathscr{C}$.

We shall also require an order-theoretic analogue of Lavrentieff's theorem (see [3]) on extending homeomorphisms to $\mathscr{G}_{-}$-sets.

Definition 9. A set $S \subset X$ is called ordinally closed (see [2], p. 128-136; [12], p. 132-134) if $S$, endowed with the relativized ordering of $X$, is a conditionally complete lattice.

Note that ordinally closed sets are $\mathscr{G}_{i}$-sets in $X$.

Lemma 5. An order isomorphism between sets $S$ and $T$ can be extended to an order isomorphism between ordinally closed sets $S^{*} \supset S$ and $T^{*} \supset T$.

Proof. We first extend the isomorphism between $S$ and $T$ to the families $\mathscr{S}$ and $\mathscr{T}$ of all ideals of $S$ and $T$, respectively, ordered by set-inclusion. Let $\mathscr{S}_{0}$ be the subfamily of $\mathscr{S}$ obtained by removing from $\mathscr{S}$ all nonprincipal ideals whose supremum relative to $S$ is an element of $S$ and let $\mathscr{T}_{0}$ be the corresponding subfamily of $\mathscr{T}$. The families $\mathscr{S}_{0}$ and $\mathscr{T}_{0}$ are isomorphic, all principal ideals of $S$ and $T$ belong to $\mathscr{S}_{0}$ and $\mathscr{T}_{0}$, respectively, and the subfamilies of principal ideals are isomorphic to $S$ and $T$. If either $S$ or $T$ is unbounded as a subset of $X$ (i.e., the supremum relative to $X$ is $+\infty$ ) then we remove from $\mathscr{S}_{0}$ and $\mathscr{T}_{0}$ their last (nonprincipal) elements. Denote by $\mathscr{S}^{*}$ and $\mathscr{T}^{*}$ the families of ideals now remaining and define $S^{*}=\left\{\sup I: I \in \mathscr{S}^{*}\right\}$ and $T^{*}=\left\{\sup J: J \in \mathscr{T}^{*}\right\}$, where the suprema are taken over $X$. The isomorphism between $\mathscr{S}^{*}$ and $\mathscr{T}^{*}$ carries over to an isomorphism between $S^{*}$ and $T^{*}$ which is an extension of the original isomorphism. Moreover the sets $S^{*}$ and $T^{*}$ are ordinally closed.

THeOREM 27. Let $(X, \mathscr{C})$ be a $\mathfrak{P}$-family such that every Borel $\mathscr{C}_{I I}$-set contains a $\mathscr{C}$-set and let $(X, \mathscr{L})$ be the $\Re$-family of Lemma 4 associated with $(X, \mathscr{C})$. If $(X, \mathscr{L})$ satisfies the condition 
(*) for ever $\mathscr{L}$-set $A$ there is an order isomorphism \& mapping $X$ onto $A$ such that $\psi(\mathscr{L})=\{B \in \mathscr{L}: B \subset A\}$,

then the absolute Baire property with respect to $(X, \mathscr{C})$ is invariant under order isomorphisms.

Proof. Assume $S$ has the absolute Baire property with respect to $(X, \mathscr{L})$ and $f$ is an order isomorphism mapping $S$ onto a set $T \subset X$. Let $\varphi$ be an order isomorphism mapping $X$ onto a set $L$. We show $T \cap L$ has the Baire property with respect to $(L, \varphi(\mathscr{L}))$.

Suppose $E$ is a $\varphi(\mathscr{L})$-set and $T \cap L$ is a $\varphi(\mathscr{L})_{I I}$-set everywhere on $E$. Apply Lemma 5 to extend $f$ to an order isomorphism $g$ between ordinally closed sets $S^{*} \supset S$ and $T^{*} \supset T$. The set $E \cap T^{*}$ being a $\mathscr{G}_{j}$-set, $\varphi^{-1}\left(E \cap T^{*}\right)$ is a Borel $\mathscr{C}_{I I^{\prime}}$-set. By hypothesis $\varphi^{-1}\left(E \cap T^{*}\right)$ contains a $\mathscr{C}$-set. Hence $E \cap T^{*}$ contains a $\varphi(\mathscr{L})$-set $F$.

Let $\psi$ be an order isomorphism mapping $X$ onto $A=\varphi^{-1}(F)$ such that $\psi(\mathscr{L})=\{B \in \mathscr{L}: B \subset A\}$ and set $h=g^{-1} \circ(\varphi \circ \psi)$. It is easily seen that $\varphi \circ \psi(\mathscr{L})=\{G \in \varphi(\mathscr{L}): G \subset F\}$ and, using Proposition 1, that every $\varphi \circ \psi(\mathscr{L})_{I}$-set $U \subset F$ is a $\varphi(\mathscr{L})_{I^{-}}$-set. Since $T \cap F$ is a $\varphi(\mathscr{L})_{I I^{-}}$ set everywhere on $F$, the set $T \cap F$ is a $\varphi \circ \psi(\mathscr{L})_{I I}$-set everywhere on $F$, and $g^{-1}(T \cap F)=S \cap h(X)$ is an $h(\mathscr{L})_{I I}$-set everywhere on $g^{-1}(F)=h(X)$. Because $S$ has the absolute Baire property with respect to $\mathscr{L}$, there is an $h(\mathscr{L})$-set $H \subset g^{-1}(F)$ such that $H \cap\left[g^{-1}(F)-\right.$ $\left.g^{-1}(T \cap F)\right]$ is an $h(\mathscr{L})_{I^{-}}$-set. Therefore $D=g(H)$ is a $\varphi \circ \psi(\mathscr{L})$-set and $D \cap[L-(T \cap L)] \subset D \cap[F-(T \cap F)]$ is a $\varphi \circ \psi(\mathscr{L})_{I}$-set. Consequently $D$ is a $\varphi(\mathscr{L})$-set contained in $E$ and $D \cap[L-(T \cap L)]$ is a $\varphi(\mathscr{L})_{I}$-set.

REMARK. Summarizing facts stated above, we see that the Marczewski sets are characterized up to equivalence among $\mathfrak{P}$-families by any one of the following properties:

1. $\mathfrak{A}(\mathscr{C})=\mathfrak{B}(\mathscr{C})$.

2. $\mathfrak{B}(\mathscr{C})$ is closed under order isomorphisms.

3. Every perfect set is a $\mathscr{C}_{I I}$-set.

8. Problems. The following questions remain unanswered, the last two of which are in the setting of [7].

1. Is the Baire property in the restricted sense invariant under order isomorphisms?

2. Does there exist an uncountable set which is a $\mathscr{C}_{I}$-set with respect to every $\mathfrak{P}$-family $(X, \mathscr{C})$ ?

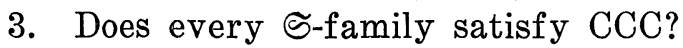

4. Is every translation invariant $\Re$-family $(X, \mathscr{C})$ satisfying CCC an 5 -family? 


\section{REFERENCES}

1. I. Bendixson, Sur la puissance des ensembles parfaits de points, Bihang till Kongl. Svenska Vetenskaps-Akademiens Handlingar, Stockholm, Band 9 (1884), Nr. 6, 15 pp.

2. G. Cantor, Contributions to the Founding of the Theory of Transfinite Numbers, translated by P.E.B. Jourdain, Dover Publ., New York.

3. M. Lavrentieff, Contribution à la théorie des ensembles homéomorphes, Fundamenta Mathematicae, 6 (1924), 149-160.

4. N. Lusin and W. Sierpiński, Sur un ensemble non dénombrable qui est de première catégorie sur tout ensemble parfait, Atti della Accademia Reale (Nazionale) dei Lincei, Rendiconti, Classe di Scienze fisiche, matematiche e naturali, Roma, Serie 6, 7 (1928), 214-215.

5. J. C. Morgan II, Infinite games and singular sets, Colloquium Mathematicum, 29 (1974), 7-17.

6. - Baire category from an abstract viewpoint, Fundamenta Mathematicae, to appear.

7. - On translation invariant families of sets, Colloquium Mathematicum, 34 (1975), 63-68.

8. J. C. Oxtoby, Measure and Category, New York 1971.

9. C. A. Rogers, Hausdorff Measures, Cambridge 1970.

10. E. Szpilrajn (Marczewski), Sur une classe de fonctions de $M$. Sierpiński et la classe correspondante d'ensembles, Fundamenta Mathematicae, 24 (1935), 17-34.

11. - O zbiorach $i$ funcjach bezwzglednie mierzalnych (On absolutely measurable sets and functions), Sprawozdania z posiedzeń Towarzystwa Naukowego Warszawskiego, Wydział III (Comptes Rendus des Séances de la Société des Sciences et des Lettres de Varsovie, Classe III), 30 (1937), 39-68.

12. W. H. Young and G. C. Young, The Theory of Sets of Points, Cambridge 1906.

Received April 19, 1976.

SYRACUSE UNIVERSITY

Current address: California State Polytechnic University, Pomona. 


\section{PACIFIC JOURNAL OF MATHEMATICS}

\section{EDITORS}

RICHARD ARENS (Managing Editor)

University of California

Los Angeles, California 90024

\section{R. A. Beaumont}

University of Washington

Seattle, Washington 98105
J. DugundjI

Department of Mathematics

University of Southern California

Los Angeles, California 90007

D. Gilbarg and J. Milgram

Stanford University

Stanford, California 94305

\section{ASSOCIATE EDITORS}
E. F. BECKENBACH
B. H. NeumanN
F. WOLF
K. YosHIDA

\section{SUPPORTING INSTITUTIONS}

\author{
UNIVERSITY OF BRITISH COLUMBIA \\ CALIFORNIA INSTITUTE OF TECHNOLOGY \\ UNIVERSITY OF CALIFORNIA \\ MONTANA STATE UNIVERSITY \\ UNIVERSITY OF NEVADA \\ NEW MEXICO STATE UNIVERSITY \\ OREGON STATE UNIVERSITY \\ UNIVERSITY OF OREGON \\ OSAKA UNIVERSITY
}

\author{
UNIVERSITY OF SOUTHERN CALIFORNIA \\ STANFORD UNIVERSITY \\ UNIVERSITY OF HAWAII \\ UNIVERSITY OF TOKYO \\ UNIVERSITY OF UTAH \\ WASHINGTON STATE UNIVERSITY \\ UNIVERSITY OF WASHINGTON \\ AMERICAN MATHEMATICAL SOCIETY
}

The Supporting Institutions listed above contribute to the cost of publication of this Journal, but they are not owners or publishers and have no responsibility for its content or policies.

Mathematical papers intended for publication in the Pacific Journal of Mathematics should be in typed form or offset-reproduced, (not dittoed), double spaced with large margins. Please do not use built up fractions in the text of your manuscript. You may however, use them in the displayed equations. Underline Greek letters in red, German in green, and script in blue. The first paragraph or two must be capable of being used separately as a synopsis of the entire paper. Items of the bibliography should not be cited there unless absolutely necessary, in which case they must be identified by author and Journal, rather than by item number. Manuscripts, in triplicate, may be sent to any one of the editors. Please classify according to the scheme of Math. Reviews, Index to Vol. 39. All other communications should be addressed to the managing editor, or Elaine Barth, University of California, Los Angeles, California, 90024.

The Pacific Journal of Mathematics expects the author's institution to pay page charges, and reserves the right to delay publication for nonpayment of charges in case of financial emergency.

100 reprints are provided free for each article, only if page charges have been substantially paid. Additional copies may be obtained at cost in multiples of 50 .

The Pacific Journal of Mathematics is issued monthly as of January 1966. Regular subscription rate: $\$ 72.00$ a year (6 Vols., 12 issues). Special rate: $\$ 36.00$ a year to individual members of supporting institutions.

Subscriptions, orders for back numbers, and changes of address should be sent to Pacific Journal of Mathematics, 103 Highland Boulevard, Berkeley, California, 94708.

PUBLISHED BY PACIFIC JOURNAL OF MATHEMATICS, A NON-PROFIT CORPORATION

Printed at Kokusai Bunken Insatsusha (International Academic Printing Co., Ltd.), 8-8, 3-chome, Takadanobaba, Shinjuku-ku, Tokyo 160, Japan. 


\section{Pacific Journal of Mathematics \\ Vol. 65, No. $2 \quad$ October, 1976}

Andrew Adler, Weak homomorphisms and invariants: an example .......... 293

Howard Anton and William J. Pervin, Separation axioms and metric-like

functions ............................................. 299

Ron C. Blei, Sidon partitions and p-Sidon sets .................... 307

T. J. Cheatham and J. R. Smith, Regular and semisimple modules ........... 315

Charles Edward Cleaver, Packing spheres in Orlicz spaces .............. 325

Le Baron O. Ferguson and Michael D. Rusk, Korovkin sets for an operator on a

space of continuous functions ............................. 337

Rudolf Fritsch, An approximation theorem for maps into Kan fibrations ....... 347

David Sexton Gilliam, Geometry and the Radon-Nikodym theorem in strict

Mackey convergence spaces .................................

William Hery, Maximal ideals in algebras of topological algebra valued

functions ...................................... 365

Alan Hopenwasser, The radical of a reflexive operator algebra ........... 375

Bruno Kramm, A characterization of Riemann algebras................. 393

Peter K. F. Kuhfittig, Fixed points of locally contractive and nonexpansive

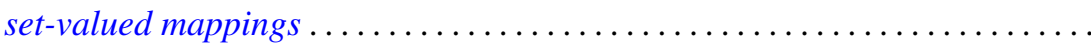

Stephen Allan McGrath, On almost everywhere convergence of Abel means of

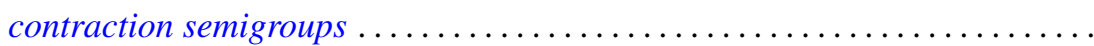

Edward Peter Merkes and Marion Wetzel, A geometric characterization of

indeterminate moment sequences............................ 409

John C. Morgan, II, The absolute Baire property ................... 421

Eli Aaron Passow and John A. Roulier, Negative theorems on generalized convex approximation .................................... 437

Louis Jackson Ratliff, Jr., A theorem on prime divisors of zero and characterizations of unmixed local domains ..............

Ellen Elizabeth Reed, A class of $T_{1}$-compactifications................... 471

Maxwell Alexander Rosenlicht, On Liouville's theory of elementary

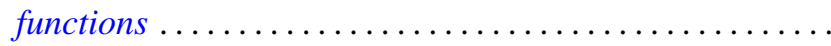

Arthur Argyle Sagle, Power-associative algebras and Riemannian

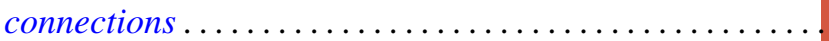

Chester Cornelius Seabury, On extending regular holomorphic maps from Stein manifolds...

Elias Sai Wan Shiu, Commutators and numerical ranges of powers of operators ...................................

Donald Mark Topkis, The structure of sublattices of the product of $n$ lattices ... 525

John Bason Wagoner, Delooping the continuous $K$-theory of a valuation

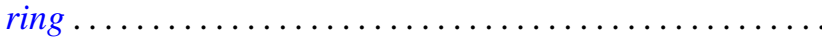

Ronson Joseph Warne, Standard regular semigroups...........

Anthony William Wickstead, The centraliser of $E \otimes_{\lambda} F \ldots$. 